NEURODEGENERATIVE DISEASE

\section{Not stuck on repeat}

Nature 546, 243-247 (2017)

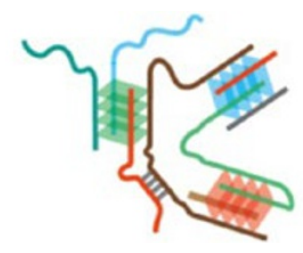

Mutations that generate nucleotide repeat expansions within RNA transcripts are common among neurodegenerative diseases such as Huntington disease (HD) and amyotrophic lateral sclerosis (ALS). Repeat-containing RNAs have been shown to accumulate aberrantly in cell nuclei as foci, leading Jain and Vale to suspect that multivalent interactions between repeatcontaining RNAs contribute to the foci and thus to disease. To test this, the authors first showed that RNA molecules containing 30 or more triplet repeats of CAG (as seen in HD) or CUG (as seen in myotonic dystrophy) could form micrometer-sized spherical clusters in vitro that associate via intermolecular base pairing and electrostatic interactions but do not require protein components. Fluorescence recovery after photobleaching experiments showed that these RNA clusters behaved as gels. In cells, similarly expanded RNAs exhibited liquidlike properties, whereby RNAs freely move in and out of the foci. The in-cell properties were likely modulated by proteins and required ATP, perhaps to remodel RNA base pairing. CAG RNA nuclear foci were sensitive to inhibitors of RNA gelation and colocalized with, but were distinct from, nuclear speckles, a reservoir of pre-mRNA splicing factors. Similar nuclear foci formed from $\mathrm{G}$-quadruplex-forming repeatcontaining RNAs associated with ALS. These results suggest that RNAs phase separate into spherical, liquid-like droplets that rapidly crosslink into gels via intermolecular base pairing and could help to explain why disease presentation only occurs after a critical number of repeats.

$M B$

\section{ENZYMOLOGY}

\section{Tracking off-targets \\ Science 356, 1084-1087 (2017)}

Inhibitors of fatty acid amide hydrolase (FAAH), which degrades amidated lipids, including anandamide, have been developed to treat pain and nervous system disorders. However, in a 2016 phase 1 trial of the FAAH inhibitor BIA 10-2474, one participant died and four others were hospitalized with neurotoxicity after receiving multiple doses of the drug. Previous clinical trials of other FAAH inhibitors reported no serious adverse events. van Esbroeck et al. tested whether off-target effects of BIA 10-2474-mediated by its covalent bonding to serine hydrolase active sites through its electrophilic imidazole urea-might account for the observed neurotoxicity. To do so, the authors incubated cells with BIA 10-2474 or a different FAAH inhibitor and treated lysates with a biotinylated fluorophosphonate probe specific for serine hydrolase active sites. Streptavidin enrichment for biotin-labeled proteins by affinity purification enabled the identification of probe-bound enzymes by MS. Putative inhibitor targets failed to bind

\section{ANTIBACTERIALS}

\section{PUMmeling RNAP}

Bacterial RNA polymerase (RNAP) is an attractive antimicrobial target, as several drug classes potently inhibit this essential protein without targeting the polymerases of mammalian hosts. Yet, the emergence of drug resistance has prompted the search for agents that are less resistance prone. Maffioli et al. now report the identification of pseudouridimycin (PUM), a natural product that inhibits RNAP by a unique mode of action. The authors identified PUM, a pseudouridine nucleoside linked to a modified Gly-GIn dipeptide, from a target-based screen of actinobacterial and fungal culture extracts. PUM inhibits bacterial RNAP activity, specifically at sites of uridine incorporation, blocks growth of Gram-positive and Gram-negative bacteria and cures infections in a mouse model. PUM has no cross-resistance with the known RNAP inhibitor rifampin, and resistance to PUM develops only one-tenth as rapidly as resistance to rifampin. Sequencing of PUM-resistant mutants identified a unique binding site for PUM, which was confirmed by a crystal structure of a transcriptional initiation complex that reveals that the pseudouridine of PUM pairs with a deoxyadenosine in the DNA template whereas the dipeptide portion engages regions that explain PUM's specificity and potency. These structural data guided the synthesis of related analogs, showing that PUM offers a new chemical scaffold and defines a unique target site for further antimicrobial development. the probe. This activity-based profiling of serine hydrolases revealed that BIA 10-2474 and its major metabolite BIA 10-2639 inhibited not only FAAH, but also lipid hydrolases and drug-metabolizing enzymes at a range of concentrations. As many of these enzymes are expressed in human brain, it's possible that the neurotoxicity observed in the BIA 10-2474 trial could be attributed to off-target inhibition resulting in altered brain lipid metabolism.

BIOCATALYSIS

\section{Achieving aminase activity}

Nat. Chem. doi: 10.1038/nchem.2783 (2017)

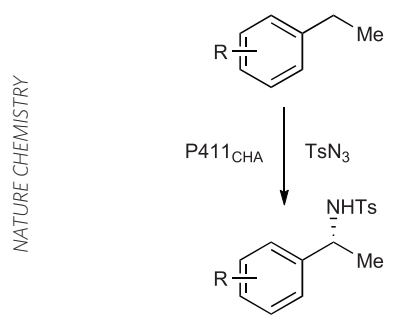

In nature, cytochrome $\mathrm{P} 450$

monooxygenases catalyze the insertion of oxygen into unactivated $\mathrm{C}-\mathrm{H}$ bonds. Downstream reactions can then convert the hydroxyl group to an amine, but no known natural enzymes exist for the direct amination of $\mathrm{C}-\mathrm{H}$ bonds. Using a hemecontaining variant of a cytochrome $\mathrm{P} 450$, termed cytochrome P411, Prier et al. used directed evolution to generate an engineered enzyme for enantioselective $\mathrm{C}-\mathrm{H}$ amination. The resulting aminase, $\mathrm{P} 411_{\mathrm{CHA}}$, uses tosyl azide $\left(\mathrm{TsN}_{3}\right)$ as a nitrene source, reacting with the heme cofactor to generate an iron nitrenoid intermediate from which the nitrene moiety is inserted into an alkane C-H bond. P411 $1_{\mathrm{CHA}}$ can catalyze up to 1,300 turnovers with $>99 \%$ enantiomeric excess in water at room temperature, under either aerobic or anaerobic conditions, making it a more efficient and environmentally friendly catalyst than existing synthetic transitionmetal complexes. Furthermore, $\mathrm{P} 411_{\mathrm{CHA}}$ is also capable of aminating diverse arenecontaining hydrocarbon substrates both as a purified enzyme and in whole Escherichia coli cells. Although $\mathrm{P} 411_{\mathrm{CHA}}$ is not able to aminate allylic or aliphatic $\mathrm{C}-\mathrm{H}$ bonds, and has limited capacity for large substrates, its development lays the foundation for future engineering of new enzymes for the selective and direct transformation of unactivated $\mathrm{C}-\mathrm{H}$ bonds.

Written by Mirella Bucci, Caitlin Deane, Alison Farrell $\&$ Terry L. Sheppard 